\title{
Reconstrucción simultánea de ambos ligamentos cruzados anteriores
}

\section{Simultaneous reconstruction of both anterior cruciate ligaments}

\author{
Muñoz-Valadez CI,* Román-Galicia J,* Juárez-Jiménez HG* \\ Instituto Mexicano del Seguro Social. Hospital General Regional Núm. 2 «Dr. Guillermo Fajardo Ortiz». Ciudad de México, México.
}

RESUMEN. Introducción: Cuando existe una lesión en ambas rodillas del ligamento cruzado anterior (LCA), el cirujano tiene la opción de reconstruir ambos de manera escalonada o simultáneamente. Existen algunos reportes y series de casos donde se realiza la reconstrucción simultanea del LCA. En este trabajo, presentamos el caso y resultado con la reconstrucción simultánea de LCA. Caso clínico: Hombre de 40 años de edad sin antecedentes de importancia. Presentaba una lesión bilateral de LCA, por lo que se le realizó cirugía sin torniquete con autoinjerto de isquiotibiales, fijado con endobutton y tornillos de interferencia. En el postoperatorio, la marcha y un programa acelerado de rehabilitación física se inició de manera inmediata. A cinco meses de seguimiento, el paciente se encuentra sin dolor y con arcos de movilidad completos y simétricos, sin complicaciones reportadas. La escala funcional de WOMAC de 35 en el preoperatorio bajó a 6 en la última consulta. Hasta el momento, la reconstrucción simultánea del LCA fue un procedimiento seguro; sin embargo, sabemos que nuestro seguimiento es muy corto para emitir alguna otra aseveración.

Palabras clave: Reconstrucción, simultánea, cruzado anterior, resultado.

\section{Introducción}

El ligamento cruzado anterior (LCA) es el encargado de limitar la traslación anterior y la rotación interna de la tibia. ${ }^{1}$ Cuando existe lesión, la reconstrucción de un ligamento roto mejora la calidad de vida de los pacientes en una ${ }^{2}$ o en ambas rodillas. ${ }^{3}$ En este último caso, el cirujano tiene la opción de reconstruir ambos ligamentos de manera escalonada o simultáneamente.
ABSTRACT. Introduction: When there is injury to both knees of the anterior cruciate ligament (ACL), the surgeon has the option to reconstruct both in a staggered manner or simultaneously. There are some reports and case series where the ACL is performed simultaneously. In this manuscript we present a case and the result with simultaneous reconstruction. Clinical case: 40 -year-old male with no history of importance with bilateral ACL injury; torniquet-free surgery was performed with autograft of hamstrings, secured with endobutton and interference screws. In the postoperative period, and an accelerated program of physical rehabilitation were immediately initiated. At five months of follow-up the patient is painless, with full, symmetrical knee range of motion. No complications reported. WOMAC's functional scale dropped from 35 preoperative to 6 in the last visit. Until now, simultaneous reconstruction of the LCA was a safe procedure; however, we know that our follow-up is too short to make any other assertions.

Keywords: Reconstruction, simultaneous, anterior cruciate, results.

\footnotetext{
* Servicio de Miembro Pélvico. Instituto Mexicano del Seguro Social. Hospital General Regional Núm. 2 «Dr. Guillermo Fajardo Ortiz». Ciudad de
} México, México.

Dirección para correspondencia:

Dr. Héctor Gerardo Juárez Jiménez

Servicio de Miembro Pélvico. Hospital General Regional Núm. 2 «Dr. Guillermo Fajardo Ortiz».

Calzada de las Bombas Núm. 117, Col. Girasoles I, C.P. 14310. Ciudad de México, México.

E-mail: hectorgerardo@gmail.com

La incidencia anual de lesión del LCA que se ha reportado en los Estados Unidos oscila entre 0.01 a $0.08 \%$, pero es mayor (1.5 a $1.7 \%$ ) entre los atletas que juegan en deportes con múltiples cambios de dirección. ${ }^{4,5}$ Por su parte, las roturas bilaterales del LCA son muy raras; la incidencia de casos bilaterales de rotura del LCA es de 2 a $4 \%$; de éstos, la mayoría no se presentan de manera simultánea, $, 6,7,8,9,10,11,12,13$ aunque existen algunos reportes y 
series de casos donde se realiza la reconstrucción simultanea del LCA, ${ }^{14,15,16,17,18,19,20}$ en los cuales se hace patente el beneficio en relación de la efectividad con el costo, el tiempo de recuperación y el no aumento en la incidencia de complicaciones.

En este trabajo, presentamos el caso y los resultados obtenidos en el corto plazo de realizar la reconstrucción del LCA de manera simultánea.

\section{Caso clínico}

Nuestro paciente es un hombre de 40 años, quien sufrió una lesión del LCA derecho tres meses previos a la lesión de la rodilla izquierda. En ambas rodillas, se lesionó con mecanismos rotacionales en actividades no deportivas (Figura 1). Fue operado tres meses posteriores a la lesión de la rodilla izquierda (seis meses de evolución de la lesión de la rodilla derecha). La intervención se realizó sin torniquete, de manera simultánea, con dos equipos quirúrgicos y dos artroscopios. En ambos procedimientos se realizó inicialmente la toma de injerto autólogo de isquiotibiales y de manera subsecuente la artroscopía.

La reconstrucción en ambos casos fue transtibial. La reconstrucción del lado derecho tardó 45 minutos; el injerto midió $8 \mathrm{~mm}$ de diámetro y fue fijado en el fémur mediante endobutton de $25 \mathrm{~mm}$ y en la tibia con un tornillo de interferencia biodegradable de $7 \mathrm{~mm}$. La reconstrucción del lado izquierdo tardó 52 minutos; el injerto midió $7 \mathrm{~mm}$ de diámetro y fue fijado con tornillos interferenciales biodegradables de $7 \mathrm{~mm}$ (Figura 2). Posterior al procedimiento, el paciente se quedó in- ternado durante tres días. De manera temprana, se inició con un programa acelerado de rehabilitación física y, al día siguiente, se indicó marcha. También se le dio antitrombótico por 28 días. Cinco semanas después, se aplicó viscosuplementación de la rodilla izquierda por persistencia de dolor.

Actualmente, el paciente tiene arcos de movilidad completos, con test de Lachman y test de cajón anterior con resultados negativos en ambas rodillas. Se aplicó la escala de WOMAC para la valoración objetiva (Tabla 1). Finalmente, el paciente se reincorporó a sus actividades habituales a los cuatro meses.

\section{Discusión}

Se sabe que realizar un procedimiento simultáneo aumenta los riesgos de complicaciones. ${ }^{21,22,23,24,25,26,27,28,29,30,31,32}$ En el caso de la reconstrucción simultánea del LCA, esto aún no ha sido documentado del todo.

Entre los reportes encontrados en la literatura, hallamos dos reportes de casos y seis series (Tabla 2). En relación con los casos, por un lado, Tifford ${ }^{8}$ no reportó los resultados de la cirugía y discutió sobre la posibilidad de una afectación previa en la escotadura intercondílea como factor predisponente para la lesión bilateral. Sajovic, ${ }^{9}$ por otro lado, reportó un caso en el cual se realizó la reconstrucción con injerto de isquiotibiales. En ese caso, el paciente operado regresó a la actividad física ocho meses después de la cirugía, además evolucionó con adecuados arcos de movilidad (5 grados de hiperextensión y 135 grados de flexión) y con una mejoría importante.
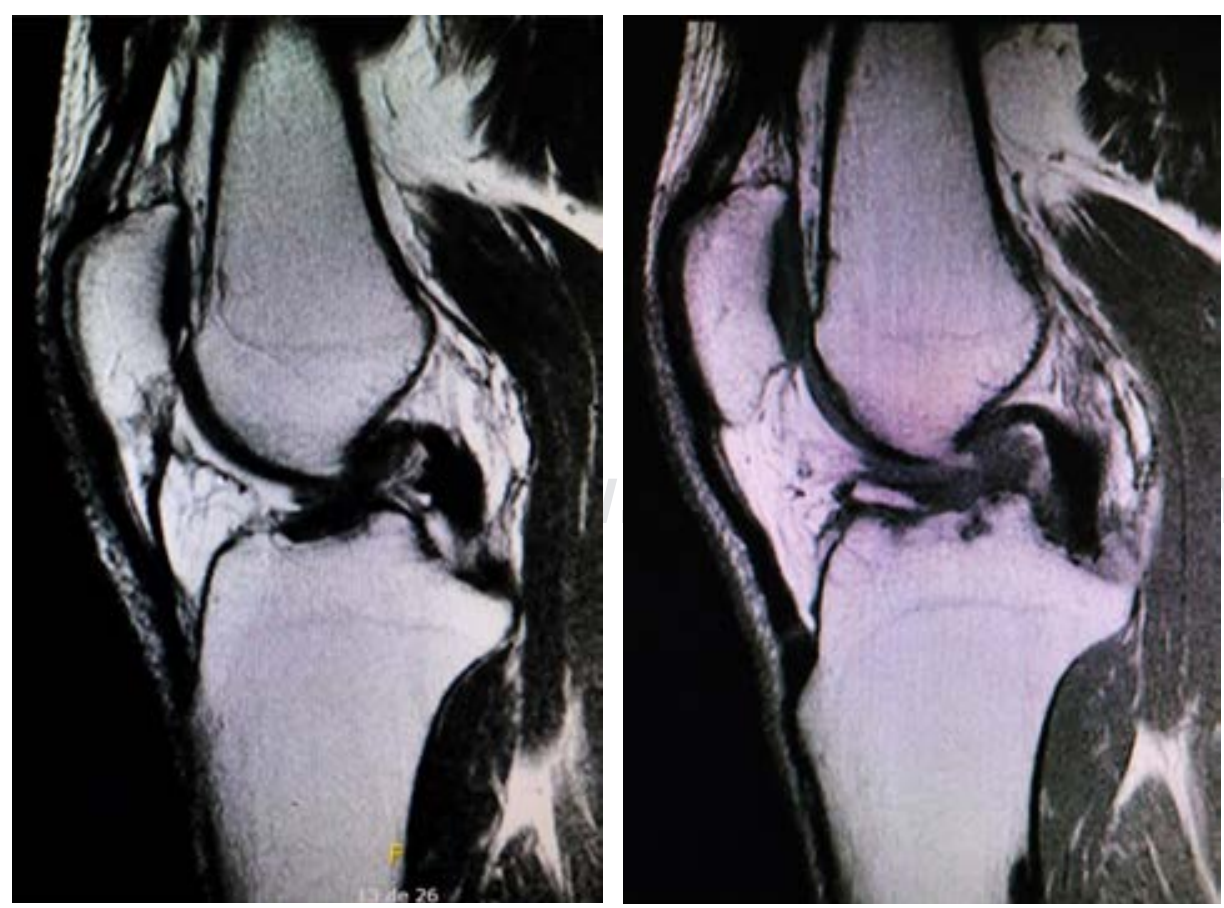

Figura 1:

Resonancia magnética preoperatoria de ambas rodillas. En el lado izquierdo, observamos la imagen de la rodilla derecha. La rodilla izquierda se muestra en la imagen del lado derecho. 

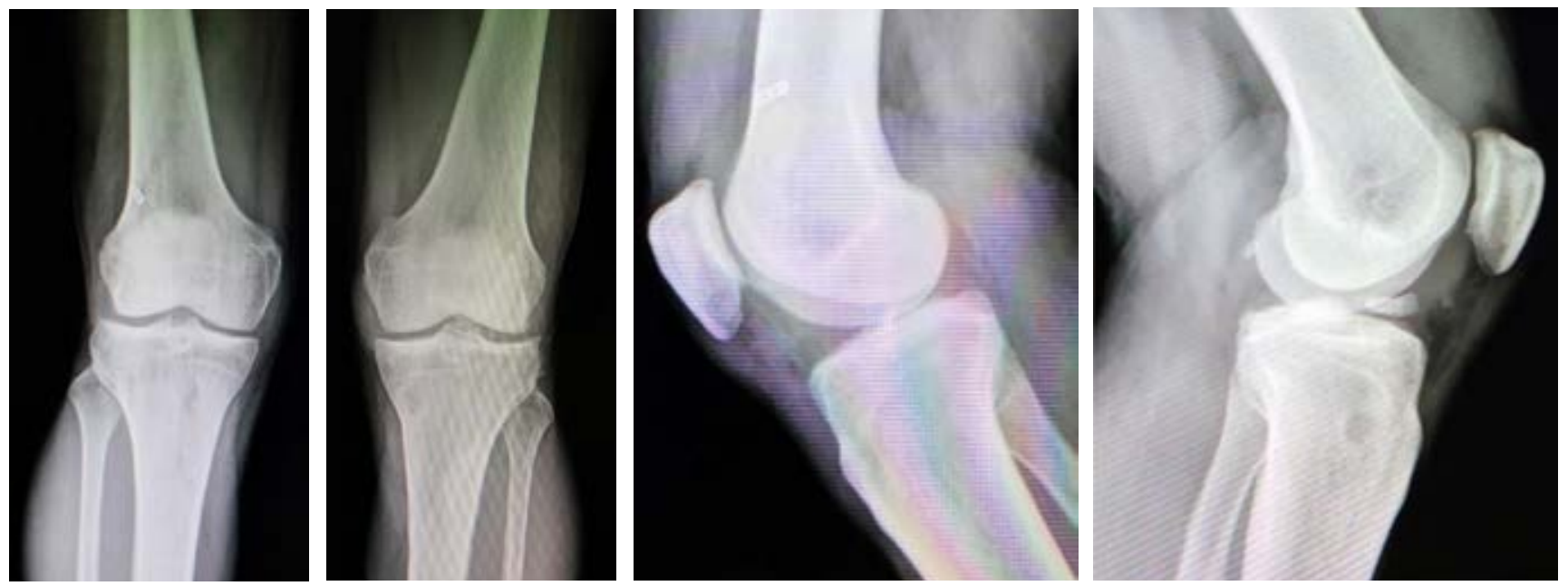

Figura 2: Radiografías postoperatorias. Sin eventualidades en la posición de los túneles en fémur y tibia.

Entre las series de casos encontradas, Hechtman ${ }^{14}$ comparó los resultados del procedimiento simultáneo con el procedimiento por estadios y reportó resultados clínicos similares. El procedimiento simultáneo fue mejor en relación costo-efectividad. El tiempo total de rehabilitación antes de regresar totalmente a la actividad deportiva fue significativamente menor en el procedimiento simultáneo. Jari y Shelbourne ${ }^{15}$ reportaron una serie de 28 pacientes, quienes se sometieron a un procedimiento simultáneo, utilizando un autoinjerto de tendón patelar; posteriormente, compararon sus resultados con un grupo control. En este estudio, reportaron que no existe diferencia significativa para el dolor postoperatorio y para los requerimientos analgésicos; aun-

Tabla 1: Resultados de la escala WOMAC en el paciente.

\begin{tabular}{|lrcc|} 
& Dolor & Rigidez & $\begin{array}{c}\text { Capacidad } \\
\text { funcional }\end{array}$ \\
\hline Preoperatorio & 11 & 4 & 35 \\
24 horas & 11 & 4 & 29 \\
48 horas & 6 & 2 & 17 \\
5 meses postoperatorio & 2 & 1 & 6 \\
\hline
\end{tabular}

que la pérdida sanguínea fue mayor en el grupo bilateral, ningún paciente requirió de transfusión. Encontraron también un regreso a la actividad laboral de tres semanas y de 6.3 meses para la inclusión total en actividades deportivas.

Larson y colaboradores ${ }^{16}$ reportaron el resultado del procedimiento simultáneo en 11 pacientes en quienes se utilizó autoinjerto de tendón patelar o aloinjerto. Demostraron que no existe un aumento en la incidencia de las complicaciones cuando se compara con un procedimiento unilateral. Saithna ${ }^{17}$ reportó ocho casos de reconstrucción bilateral: siete con isquiotibiales y uno con tendón patelar. Con 28 meses de seguimiento, no encontró complicaciones como infección, tromboembolismo o falla del injerto. Sajovic ${ }^{18}$ reportó siete casos en quienes se realizó la reconstrucción de manera simultánea. Se reportó un regreso a las actividades físicas de nueve meses. En todos los casos se utilizó torniquete y, de igual manera, no se observó aumento en los riesgos. El costo total del procedimiento fue menor que en la realización del procedimiento por estadios.

Panigrahi ${ }^{19}$ realizó un estudio multicéntrico prospectivo en el cual se hicieron 14 cirugías para la reconstrucción simultánea de LCA con injerto autólogo de isquiotibiales fijado en fémur con endobutton y en tibia con un tornillo

Tabla 2: Literatura que reporta los resultados de la reconstrucción simultánea del ligamento cruzado anterior.

\begin{tabular}{|c|c|c|c|c|}
\hline Autor & Año & País & Número de casos & Período \\
\hline Hechtman & 1998 & Florida, Estados Unidos & 7 & 1984 а 1992 (8 años) \\
\hline Tifford & 2001 & California, Estados Unidos & 1 & \\
\hline Jari & 2000 & Indianápolis, Estados Unidos & 28 & 1995 a 1999 (4 años) \\
\hline Larson & 2004 & Minneapolis, Estados Unidos & 11 & 1993 a 2001 (8 años) \\
\hline Saithna & 2010 & Coventry, Reino Unido & 8 & No se menciona \\
\hline Sajovic & 2013 & Celje, Eslovenia & 7 & 2002 a 2008 (6 años) \\
\hline Panigrahi & 2015 & Odisha, India & 14 & 2010 a 2014 (4 años) \\
\hline Vaishya & 2018 & New Delhi, India & 5 & No se menciona \\
\hline
\end{tabular}




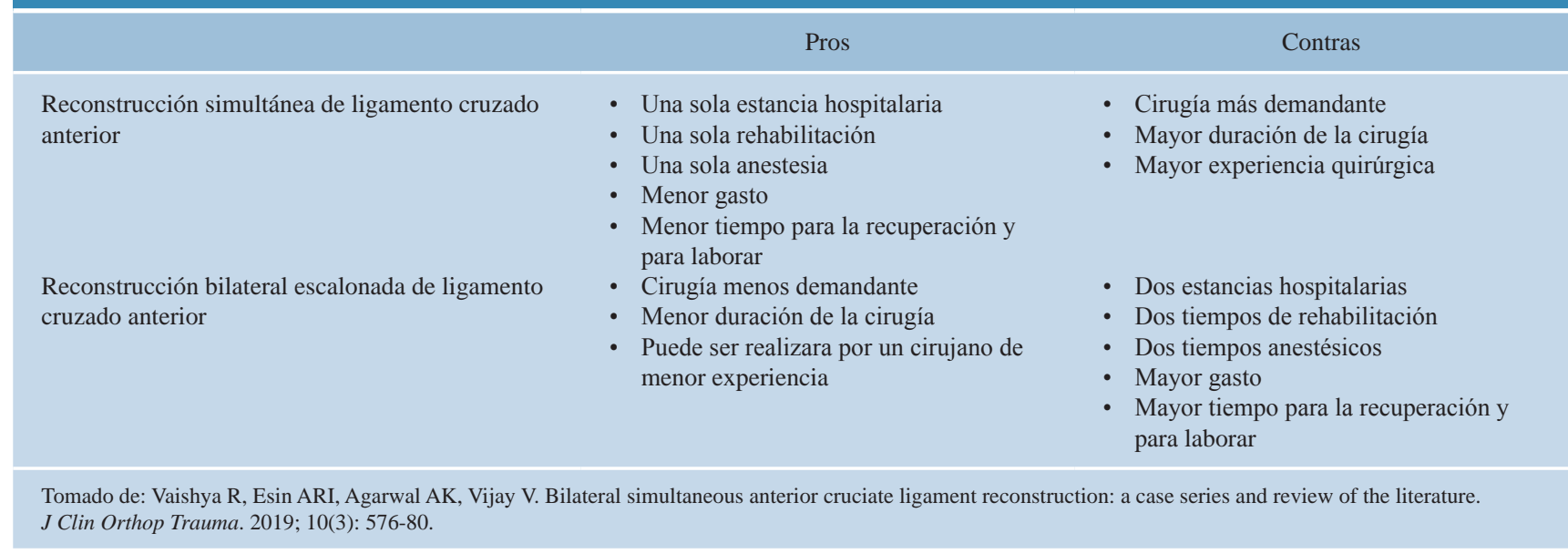

de interferencia. El seguimiento promedio fue de 28 meses (24 a 38 meses). Este autor concluyó que la reconstrucción simultánea es un procedimiento favorecedor desde el punto de vista del costo-beneficio.

Vaishya ${ }^{20}$ reportó cinco pacientes en los cuales se realizó la reconstrucción con injerto de isquiotibiales. En su seguimiento de 3.2 años en promedio, se observó una adecuada evolución.

La realización del procedimiento de reconstrucción de manera simultánea es actualmente un debate para nosotros. En una encuesta realizada por Saadat $\mathrm{E}^{33}$ se observó que la mayoría de los encuestados preferían realizar una reconstrucción no simultánea del ligamento cruzado anterior, a pesar de la evidencia al momento del estudio de que la reconstrucción simultánea no implica mayores riesgos. En nuestro caso, no encontramos complicaciones como síndrome compartimental, algún evento trombótico, fallo del injerto o infección. La evidencia actual no muestra mayores riesgos en la realización de la reconstrucción simultánea del LCA que cuando se realiza por estadios (Tabla 3); tampoco hay diferencia cuando se compara con la reconstrucción unilateral. Es importante documentar el seguimiento de una cohorte prospectiva para mejorar la evidencia al respecto.

Hasta el momento, en nuestro caso con cinco meses de seguimiento, la reconstrucción simultánea del LCA ha demostrado ser un procedimiento seguro; en la literatura revisada, se prefiere el uso de autoinjerto de isquiotibiales sobre otros. Sin embargo, sabemos que nuestro seguimiento es muy corto como para emitir alguna otra aseveración.

Referencias

1. Zantop T, Peterson W, Fu FH. Anatomy of the anterior cruciate ligament. Oper Tech Orthop. 2005; 15: 20-8.

2. Filbay SR. Longer-term quality of life following ACL injury and reconstruction. Br J Sports Med. 2018; 52: 208-9.
3. Ristić V, Ristić S, Maljanović M, Milankov V, Harhaji V, Đuričin A. Quality of life after bilateral anterior cruciate ligament reconstructions. Med Pregl. 2015; 68: 308-15.

4. Vaishya R, Agarwal AK, Ingole S, Vijay V. Current trends in anterior cruciate ligament reconstruction: a review. Cureus. 2015; 7: e378.

5. Huston LJ, Greenfield ML, Wojtys EM, Griffin LY, Garrick JG. Anterior cruciate ligament injuries in the female athlete: potential risk factors. Clin Orthop Relat Res. 2000; 372: 50-63.

6. Maywood RM, Hechtman KS. Simultaneous bilateral anterior cruciate ligament tears. Am J Knee Surg. 1995; 8: 134-6.

7. Sanchis-Alfonso V, Tintó-Pedrerol M. Simultaneous bilateral anterior cruciate ligament tears in a female beginner skier. Knee Surg Sports Traumatol Arthrosc. 2000; 8: 241-3.

8. Tifford CD, Jackson DW. Simultaneous bilateral anterior cruciate ligament ruptures in a cheerleader. Arthroscopy. 2001; 17: E17.

9. Sajovic M, Demsar S. One-stage bilateral anterior cruciate ligament reconstruction with use of hamstring tendon autografts: a case report. Knee Surg Sports Traumatol Arthrosc. 2007; 15: 1114-5.

10. Souryal TO, Moore HA, Evans JP. Bilaterality in anterior cruciate ligament injuries: associated intercondylar notch stenosis. Am J Sports Med. 1988; 16: 449-54.

11. Anderson AF, Lipscomb AB, Liudah KJ, Addlestone RB. Analysis of the intercondylar notch by computed tomography. Am J Sports Med. 1987; 15: 547-52.

12. Kvist J, Kartus J, Karlsson J, Forssblad M. Results from Swedish national anterior cruciate ligament register. Arthroscopy. 2014; 30: 803-10.

13. Ristić V, Ristić S, Maljanović M, Đan V, Milankov V, Harhaji V. Risk factors for bilateral anterior cruciate ligament injuries. Med Pregl. 2015; 68: 192-7.

14. Hechtman KS, Tjin-Tsoi EW, Uribe JW, Kessler K, Vargas LA, Gables C. Simultaneous vs staged bilateral anterior cruciate ligament reconstruction with endoscopic technique. Arthroscopy. 1998; 14: S17.

15. Jari S, Shelbourne KD. Simultaneous bilateral anterior cruciate ligament reconstruction. Am J Sports Med. 2000; 30: 891-5.

16. Larson CM, Fischer DA, Smith JP, Boyd JL. Bilateral anterior cruciate ligament reconstruction as a single procedure: evaluation of cost and early functional results. Am J Sports Med. 2004; 32: 192-200.

17. Saithna A, Arbuthnot J, Carey-Smith R, Spalding T. Simultaneous bilateral anterior cruciate ligament reconstruction: a safe option. Knee Surg Sports Traumatol Arthrosc. 2010; 18: 1071-4.

18. Sajovic M, Demsar S, Sajovic R. One-stage bilateral anterior cruciate ligament reconstruction. Knee Surg Sports Traumatol Arthrosc. 2013; 21: 1998-2003.

19. Panigrahi R, Mahapatra AK, Priyadarshi A, Palo N, Biswal MR. Bilateral ACL reconstructions with hamstring autografts. J Knee Surg. 2016; 29: 403-8. 
20. Vaishya R, Esin ARI, Agarwal AK, Vijay V. Bilateral simultaneous anterior cruciate ligament reconstruction: a case series and review of the literature. J Clin Orthop Trauma. 2019; 10(3): 576-80.

21. Bierbaum BE, Callaghan JJ, Galante JO, Rubash HE, Tooms RE, Welch RB. An analysis of blood management in patients having a total hip or knee arthroplasty. J Bone Joint Surg. 1999; 81A: 2-10.

22. Lane GJ, Hozack WJ, Shah S, Rothman RH, Booth RE Jr, Eng $\mathrm{K}$, et al. Simultaneous bilateral versus unilateral total knee arthroplasty. Outcomes analysis. Clin Orthop Relat Res. 1997; 345: 106-12.

23. Dorr LD, Merkel C, Mellman MF, Klein I. Fat emboli in bilateral total knee arthroplasty. Predictive factors for neurologic manifestations. Clin Orthop Relat Res. 1989; 248: 112-8.

24. Ries MD. Bilateral total knee replacement is relatively safe but carries risks. Orthopedics. 1998; 21: 414-6.

25. Vince KG. Bilateral total knee arthroplasty under one anesthesia: a safe protocol. Mayo Clin Proc. 1997; 72: 883-5.

26. Alfaro-Adrian J, Bayona F, Rech JA, Murray DW. One- or two-stage bilateral total hip replacement. J Arthroplasty. 1999; 14: 439-45.
27. Lorenze M, Huo MH, Zatorski LE, Keggi KJ. A comparison of the cost effectiveness of one-stage versus two-stage bilateral total hip replacement. Orthopedics. 1998; 21: 1249-52.

28. Ritter MA, Randolph JC. Bilateral total hip arthroplasty: a simultaneous procedure. Acta Orthop Scand. 1976; 47: 203-8.

29. Cohen RG, Forrest CJ, Benjamin JB. Safety and efficacy of bilateral total knee arthroplasty. J Arthroplasty. 1997; 12: 497-502.

30. Hardaker WT Jr, Ogden WS, Musgrave RE, Goldner JL. Simultaneous and staged bilateral total knee arthroplasty. J Bone Joint Surg. 1978; 60A: 247-50.

31. Reuben JD, Meyers SJ, Cox DD, Elliott M, Watson M, Shim SD. Cost comparison between bilateral simultaneous, staged, and unilateral joint arthroplasty. J Arthroplasty. 1998; 13: 172-9.

32. Mutsuzaki H, Watanabe A, Komatsuzaki T, Kinugasa T, Ikeda K. Investigation of perioperative safety and clinical results of one-stage bilateral total knee arthroplasty in selected low-risk patients. J Orthop Surg Res. 2018; 17: 14.

33. Saadat E, Curry EJ, Li X, Matzkin EG. Bilateral simultaneous anterior cruciate ligament injury: a case report and national survey of orthopedic surgeon management preference. Orthop Rev (Pavia). 2014; 6: 5551. 